\title{
mySinusitisCoach: patient empowerment in chronic rhinosinusitis using mobile technology*
}

\author{
S.F. Seys ${ }^{1,2}$, J. Bousquet ${ }^{3}$, C. Bachert ${ }^{4,5}$, W.J. Fokkens ${ }^{6}$, I. Agache', M. Bernal- \\ Sprekelsen ${ }^{8}$, I. Callebaut ${ }^{9}$, L.O. Cardel ${ }^{10}$, S. Carrie $^{11}$, P. Castelnuovo ${ }^{12}$, \\ R. Cathcart ${ }^{13}$, J. Constantinidis ${ }^{14}$, L. Cools ${ }^{9}$, M. Cornet ${ }^{6}$, G. Clement ${ }^{15}$, \\ J.C. de Sousa ${ }^{16}$, T. Cox ${ }^{17}$, M. Doulaptsii ${ }^{18}$, P. Gevaert ${ }^{4}$, C. Hopkins ${ }^{19}$, V. Hox ${ }^{20}$, \\ T. Hummel ${ }^{21}$, W. Hosemann ${ }^{22}$, R. Jacobs ${ }^{23}$, M. Jorissen', B.N. Landis ${ }^{24}$, \\ A. Leunig ${ }^{25}$, V.J. Lund ${ }^{26}$, J. Mullol27, M. Onerci ${ }^{28}$, S. Palkonen ${ }^{29}$, I. Proano ${ }^{29}$, \\ E. Prokopakis ${ }^{17}$, D. Ryan ${ }^{30}$, H. Riechelmann ${ }^{31}$, J. Saevels ${ }^{32}$, C. Segboer 6 , \\ K. Speleman ${ }^{33}$, E.A. Steinsvik ${ }^{34}$, P. Surda ${ }^{18}$, P.V. Tomazic ${ }^{35}$, O. Vanderveken ${ }^{36}$, \\ L. Van Gerven ${ }^{9}$, T. Van Zele ${ }^{4}$, B. Verhaeghe ${ }^{37}$, K. Vierstraete ${ }^{38}$, S. Vlaminck ${ }^{33}$, \\ J. Wilkinson ${ }^{39}$, S. Williams ${ }^{40}$, B. Pugin 1,2, P.W. Hellings s,2,9 $^{1}$
}

Rhinology 56: 3, 209-215, 2018 https://doi.org/10.4193/Rhin17.253

*Received for publication: December 19, 2017 Accepted: February 1, 2018

' European Forum for Research and Education in Allergy and Airway Diseases (EUFOREA), Brussels, Belgium

${ }^{2}$ Laboratory of Clinical Immunology, Department of Microbiology and Immunology, KU Leuven, Belgium

${ }^{3}$ Department of Respiratory Disease, University Hospital Arnaud de Villeneuve, Montpellier, France

${ }^{4}$ Upper Airways Research Laboratory, Department of Otorhinolaryngology-Head and Neck Surgery, Ghent University, Belgium

${ }^{5}$ Department of Otorhinolaryngology, Karolinska Institute, Stockholm, Sweden

${ }^{6}$ Department of Otorhinolaryngology, Academic Medical Center, Amsterdam, The Netherlands

${ }^{7}$ Faculty of Medicine, Transylvania University, Brasov, Romania

${ }^{8}$ Department of Otorhinolaryngology, Hospital Clínic Universitari, Barcelona, Spain

${ }^{9}$ Department of Otorhinolaryngology-Head and Neck Surgery, UZ Leuven, Belgium

${ }^{10}$ Division of ENT Diseases, Department of Clinical Science, Intervention and Technology, Karolinska Institutet, Stockholm, Sweden

${ }^{11}$ ENT Department, Freeman Hospital, Newcastle upon Tyne, UK

${ }^{12}$ ENT Department, Ospedale Di Circolo E Fondazione Macchi, Varese, Italy

${ }^{13}$ ENT Department, Cumberland Infirmary, Carlisle, Cumbria, UK

${ }^{14}$ 2nd Academic ENT department, Aristotle University of Thessaloniki, Papageorgiou General Hospital, Thessaloniki, Greece

${ }^{15}$ ENT Department, AZ Damiaan, Oostende, Belgium

${ }^{16}$ Community Health, Life and Health Sciences Research Institute, School of Health Sciences, Univerity of Minho, Portugal

${ }^{17}$ ENT Department, Jessa hospital, Hasselt, Belgium

${ }^{18}$ Department of Otorhinolaryngology, University of Crete School of Medicine, Heraklion, Crete, Greece

${ }^{19}$ ENT Department, Guy's and St Thomas' NHS Foundation Trust, London, United Kingdom

${ }^{20}$ Département d'Otorhinolaryngologie, Cliniques Universitaires Saint-Luc, Belgium

${ }^{21}$ Smell and Taste Clinic, ENT Department, Technische Universität Dresden, Dresden, Germany

${ }^{22}$ ENT Department, University of Greifswald, Germany

${ }^{23}$ Department of Otorhinolaryngology - Head and Neck Surgery, General Hospital Sint-Blasius, Dendermonde, Belgium

${ }^{24}$ Unité de Rhinologie-Olfactologie, Service d'Oto-Rhino-Laryngologie et de Chirurgie cervico-faciale, Hôpitaux Universitaires de Genève, Genève,

Suisse

${ }^{25}$ ENT Department, Ludwig Maximilians University Munich, Germany

${ }^{26}$ UCL \& Honorary Consultant ENT Surgeon, UCLH, UK

${ }^{27}$ IDIBAPS, Hospital Clínic, Universitat de Barcelona, CIBERES. Barcelona, Catalonia, Spain

${ }^{28}$ ENT Department, Faculty of Medicine, Hacettepe University, Ankara, Turkey

${ }^{29}$ European Federation of Allergy and Airways Diseases Patients' Associations (EFA), Brussels, Belgium 


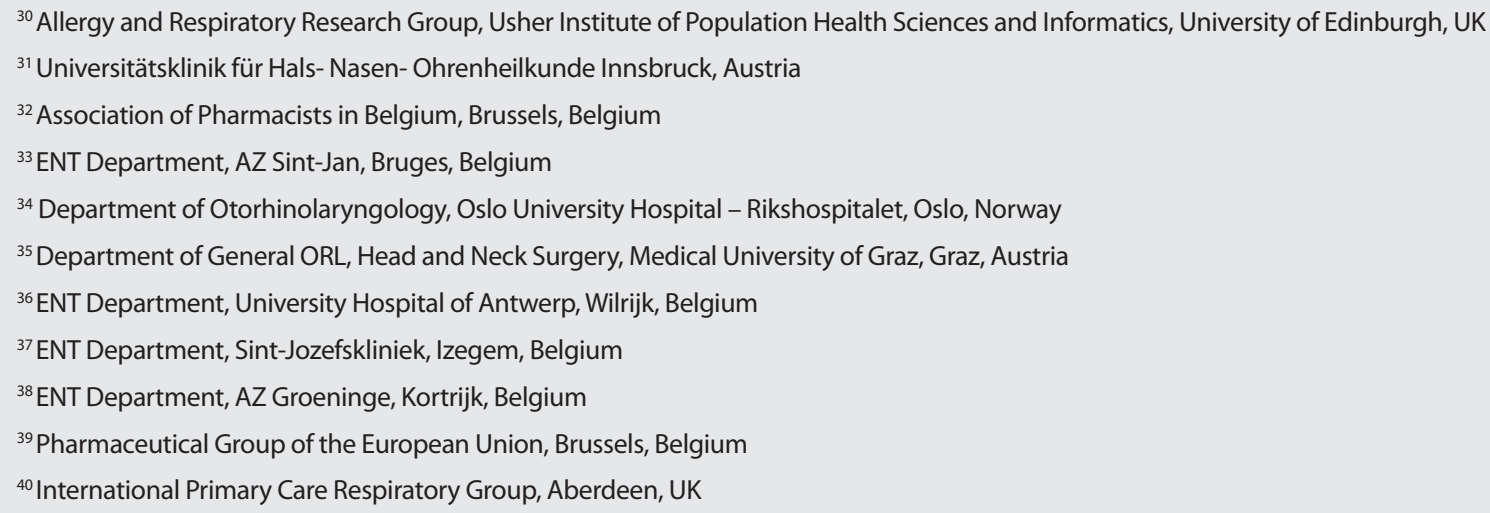

\begin{abstract}
Mobile health technology is emerging to take a prominent position in the management of chronic diseases. These technologies aim at enhancing patient empowerment via education and self-management. To date, of all the different apps available for patients with sinus disease, none were developed by medical experts dealing with chronic rhinosinusitis (CRS). The European Forum for Research and Education in Allergy and Airway diseases (EUFOREA) has undertaken a multi-stakeholder approach for designing, developing and implementing a tool to support CRS patients in monitoring their symptoms and to provide patients with a digital support platform containing reliable medical information about their disease and treatment options. mySinusitisCoach has been developed by medical experts dealing with CRS in close collaboration with patients, primary care physicians and community pharmacists, meeting the needs of both patients and health care providers. From a research perspective, the generation of real life data will help to validate clinical studies, patient stratification and improve understanding of the socio-economic impact of CRS, thereby paving the way for better treatment strategies.
\end{abstract}

Key words: chronic airway disease, rhinosinusitis, mobile application, education, self-management

\section{Introduction}

Chronic rhinosinusitis (CRS) affects $11 \%$ of the European population and is associated with a significant socio-economic burden $(1,2)$. CRS with and without nasal polyps are the main constituent disease entities with each having different pathophysiological mechanisms or endotypes underlying the disease ${ }^{(3)}$. Evidence-based treatment guidelines (EPOS) provide guidance to physicians for the management of CRS aiming at achieving or maintaining clinical control ${ }^{(4)}$. The cornerstone of CRS treatment is prolonged anti-inflammatory treatment in combination with Functional Endoscopic Sinus Surgery (FESS) in case of failure of medical treatment. Despite currently available treatment modalities for CRS, including sinus surgery, up to $40 \%$ of patients still suffer from uncontrolled disease ${ }^{(5)}$. Few studies so far have evaluated the burden of uncontrolled CRS in real life using the EPOS criteria for CRS disease control ${ }^{(5,6)}$.

Uncontrolled upper airway disease might be attributed to disease-, treatment-, diagnosis- and patient-related factors (7). The relative importance of these factors still needs to be determined but it has been suggested that active participation of patients in the management of their disease may reduce the burden of uncontrolled disease through better adherence and personalized care ${ }^{(8,9)}$. Mobile health technology has the potential to increase patient participation via self-management and education. Recently, education and personalized messages were identified in market research by app developers as most effective in changing patient behavior and are easy to implement ${ }^{(10)}$. More challenging, but still having a high impact are app features that improve the communication with the healthcare provider ${ }^{(10)}$. The success of future mobile ( $m$ )Health tools likely depends on the inclusion of those features that are lacking in most currently available apps for chronic upper airway diseases.

For example, MASK-rhinitis is a pioneering project allowing allergic rhinitis patients to track their nasal, ocular or lung symptoms and share it with their health care provider ${ }^{(11,12)}$. First results demonstrated its utility to phenotype patients with allergic rhinitis (AR) and to evaluate the impact of allergic rhinitis on work productivity ${ }^{(13,14)}$. Recently, the use of a visual analogue scale (VAS) on smartphone screens was validated to assess AR control ${ }^{(15)}$. Follow-up studies are needed to demonstrate its utility in 
improving disease control and adherence to treatment by the use of the app. Currently, different mobile apps related to sinus disease are available but none were developed by medical experts dealing with CRS. EUFOREA started a program to increase patient participation and empowerment in CRS, encouraging them to become active players in the integrated care pathway ${ }^{(16)}$. This approach fully matches the concept of precision medicine, for which participation of the patient is one of the 4 key pillars ${ }^{(9,17)}$. mySinusitisCoach has been developed in collaboration with the EUFOREA network of Rhinology experts, and different national and international bodies representing patients, primary care physicians, pharmacists and the eHealth community. This manuscript describes the developmental process, the different functionalities and the expected benefits of the app. We here aim to inform the Rhinology community about the unique approach used to design, develop and implement the app, which might serve as a state-of-the-art example for other projects.

\section{Identifying the needs in CRS management}

The needs of patients with CRS vary depending on the phase of their disease severity, experience and geographical distribution. It is assumed that patients with upper airway symptoms rely on self-medication and/or over-the-counter treatment to deal with the first symptoms of rhinosinusitis. Early detection of CRS is warranted to avoid delay in presentation to the primary care physician or ENT specialist. An integrated approach aligning different health care providers dealing with CRS is needed to deliver a uniform message to the patient ${ }^{(9)}$. While newly diagnosed patients need reliable information about their disease and treatment options, patients with established or uncontrolled disease also require closer follow-up of their symptoms and disease control. Additionally, patients undergoing surgery will benefit from intensified monitoring of their symptoms. From the research perspective, there is a need to validate findings from clinical studies with real life data to guarantee applicability at the population level ${ }^{(18)}$.

\section{Implementation of supported self-management into clinical practice in CRS}

Treatment algorithms have been developed and presented in EPOS guidelines ${ }^{(4)}$. From the patient's perspective, poor adherence to treatment, incorrect use of treatment and limited knowledge about CRS and the treatment options might hamper their path to maintain or achieve optimal disease control ${ }^{(19-21)}$. A blended health care approach is needed where the patient is well informed about the treatment options by the physician, in accordance with the guidelines, allowing the patient to understand the rationale of a treatment decision. Mobile health tools are aimed at supporting this process and allow disease monitoring over time, which contributes to supported selfmanagement of the disease.

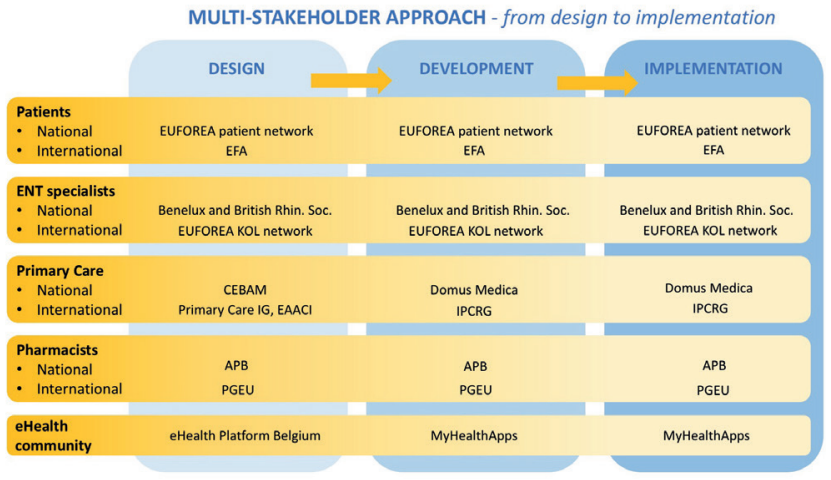

Figure 1. Multi-stakeholder approach from design to implementation. EFA: European Federation of Allergy and Airways Diseases Patients' Associations, CEBAM: Belgian Centre for Evidence-Based Medicine, Domus Medica: Belgian Association of Primary Care Physicians, EAACl: European Academy of Allergy and Clinical Immunology, IPCRG: International Primary Care Group, APB: Association of Pharmacists in Belgium, PGEU: Pharmaceutical Group of the European Union.

\section{Development of mySinusitisCoach: a multi-stake- holder approach from design to implementation}

EUFOREA aimed at consulting all relevant stakeholders early on during the process of design and development of mySinusitisCoach. Patient-focused group meetings were organized in different countries throughout Europe to identify the patients' needs and their perspective on new developments in the field of mobile health technology. In addition, national as well as international representatives of patient organizations (EFA), associations of primary care physicians (Domus Medica, IPCRG), associations of community pharmacists (Association of Pharmacists in Belgium or APB and Pharmaceutical Group of the EU or PGEU) and E-Health community have provided feedback on design and development of mySinusitisCoach (Figure 1). This approach creates a unique European platform for dissemination of mySinusitisCoach and for implementation into clinical practice.

\section{mySinusitisCoach: availability and data protection}

The system has been initially deployed in three countries (Belgium, The Netherlands and United Kingdom) and is available in two languages (Dutch and English). The app can be downloaded for free from the app stores (for iPhone: App Store, for smartphones that run on Android: Google Play). mySinusitisCoach has been registered as a class I medical device. Data aggregated in an anonymized way through mySinusitisCoach are stored on a private, secured server (ISO 27001 certified) for '15 years. A penetration test for the backend website of mySinusitisCoach is scheduled for 2018. Geolocalized data is only stored after explicit consent by the app user. The data are owned by EUFOREA. The Terms of Use allow the use of anonymized data for research purposes. 

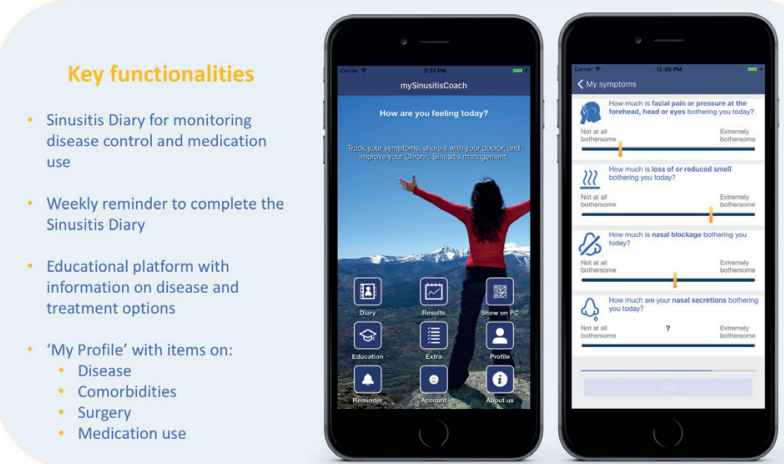

Figure 2. Key functionalities of mySinusitisCoach.

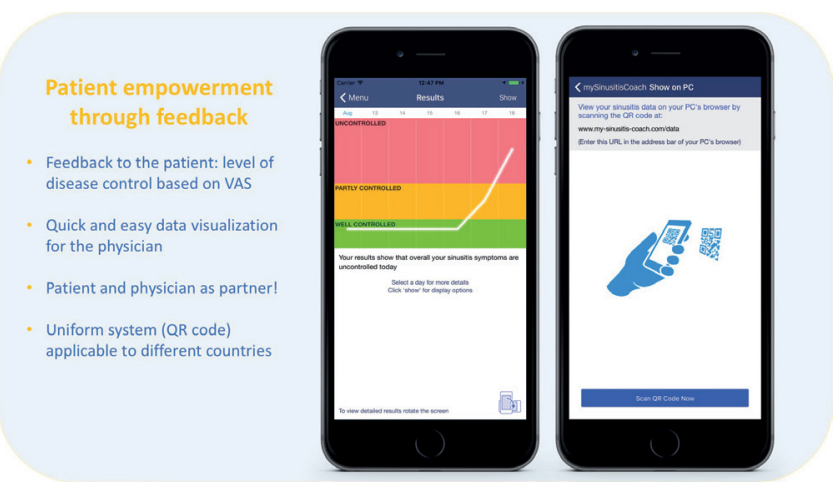

Figure 3. Patient empowerment through feedback to the patient and the physician.

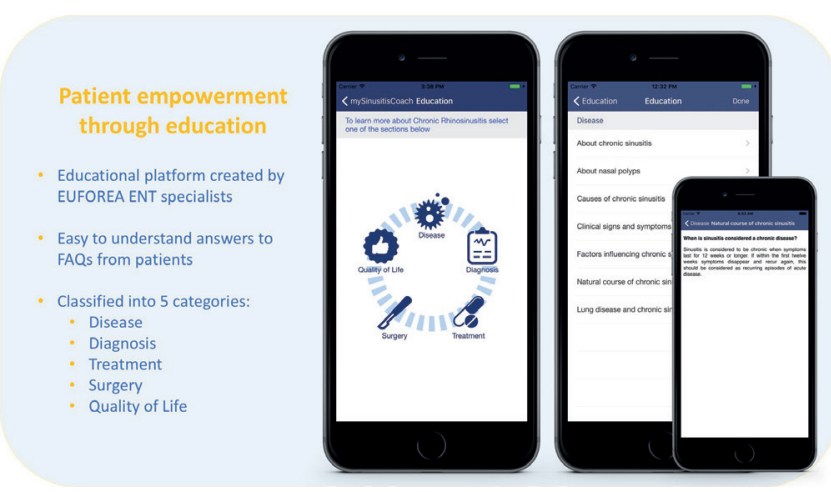

Figure 4. Patient empowerment via patient education.

\section{Monitoring disease control}

\section{Profile of the patient}

Demographic characteristics (year of birth, gender, country, language, smoking status), presence of co-morbidities (AR, asthma, COPD), disease-related factors (duration of symptoms, presence of nasal polyps, previous surgery) are recorded in the 'Profile' menu by the patient themselves. Country-specific medication lists for nasal, lung or eye symptoms are available from the 'Profile' menu and allow patients to indicate their medication use, for which compliance can be recorded after filling the Diary.
Table 1. Sinusitis Diary questions.

\begin{tabular}{|c|c|}
\hline VAS for & Question \\
\hline Global sinusitis symptoms & $\begin{array}{l}\text { How much are your global sinusitis } \\
\text { symptoms bothering you today? }\end{array}$ \\
\hline Facial pain or headache & $\begin{array}{l}\text { How much is facial pain or pressure at } \\
\text { the forehead, head or eyes bothering you } \\
\text { today? }\end{array}$ \\
\hline Smell impairment & $\begin{array}{l}\text { How much is loss of or reduced smell } \\
\text { bothering you today? }\end{array}$ \\
\hline Nasal Blockage & $\begin{array}{l}\text { How much is nasal blockage bothering } \\
\text { you today? }\end{array}$ \\
\hline Nasal secretions & $\begin{array}{l}\text { How much are your nasal secretions } \\
\text { bothering you today? }\end{array}$ \\
\hline Post-nasal drip & $\begin{array}{l}\text { How much are nasal secretions dripping } \\
\text { into the throat bothering you today? }\end{array}$ \\
\hline Lower airway symptoms & $\begin{array}{l}\text { How much is shortness of breath or } \\
\text { wheezing bothering you today? }\end{array}$ \\
\hline Impact on sleep quality & $\begin{array}{l}\text { How much are your sinusitis symptoms } \\
\text { affecting your sleep quality? }\end{array}$ \\
\hline $\begin{array}{l}\text { Impact on work and daily } \\
\text { life }\end{array}$ & $\begin{array}{l}\text { How much are your sinusitis symptoms } \\
\text { affecting your work or daily activities } \\
\text { today? }\end{array}$ \\
\hline
\end{tabular}

\section{Sinusitis Diary}

The Sinusitis Diary is a simple tool to monitor rhinosinusitis symptoms and their impact on lower airway symptoms, sleep quality and work or daily life. This tool relies on a visual analogue scale, allowing the patient to score how bothersome a particular symptom is for him/her on a particular day. A similar system has been used and validated in the past to monitor allergy symptoms ${ }^{(15,22)}$. The questions of the Sinusitis Diary are listed in Table 1. Questions with a previous VAS $\leq 2$ (e.g. result of the day before) were dropped from the Diary for 1 week to avoid an overload of questions that might be irrelevant for the patient at that time.

In addition to rhinosinusitis symptoms, medication use is recorded as a last item of the Diary. A weekly reminder to complete the Diary is activated in the app. This can be switched off/on and the time point can be chosen according to the patients' preference.

\section{Feedback to the patient}

Upon completion of the Sinusitis Diary, the patient receives feedback about the level of control of his/her global rhinosinusitis symptoms: well controlled (VAS $\leq 2$ ), partly controlled (VAS $>2$ and VAS $\leq 5)$, uncontrolled $(V A S>5){ }^{(5)}$. The results of the specific symptoms over time are visualized when rotating the screen of the mobile device into landscape mode. A patient who has uncontrolled disease for a prolonged time receives the following notifications:

- $\quad$ After 1 week: 'Your rhinosinusitis symptoms are uncontrolled. Did you properly rinse your nose with saline?' 


\section{Expected benefits of mySinusitisCoach}

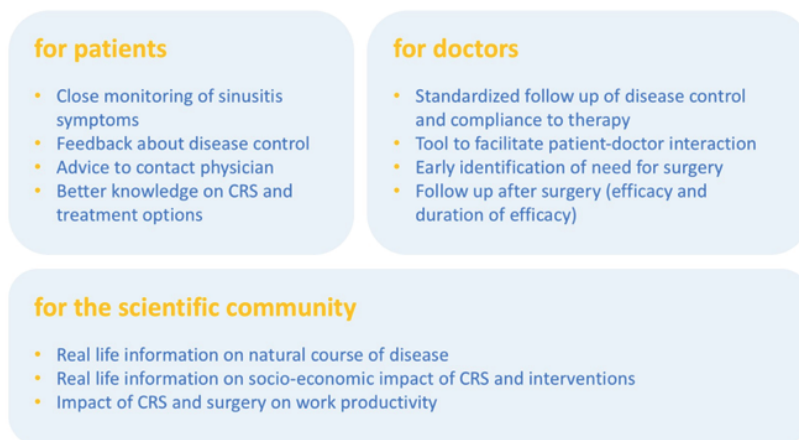

Figure 5. Expected benefits of mySinusitiscoach for the different stakeholders.

- After 2 weeks: 'Your rhinosinusitis symptoms are uncontrolled for 1 week. Did you properly take your medication?'

- $\quad$ After 3 weeks: 'Your sinusitis symptoms are uncontrolled for 2 weeks. Uncontrolled sinus disease is seen in $40 \%$ of patients with chronic rhinosinusitis who were prescribed nasal sprays and have had sinus surgery. The reasons for that might be due to exposure to air pollution or allergens, other airway diseases, medication that is inefficiently reducing the ongoing inflammation or not taking adequately your medication.'

- $\quad$ After 4 weeks: 'Your rhinosinusitis symptoms are uncontrolled for 4 weeks. You may consider visiting your GP or ENT doctor to discuss your symptoms and check your treatment.'

\section{Feedback to the physician}

During patient consultation, the data on the level of disease control, VAS of specific symptoms and use of medication can be visualized on any PC with internet connection. The link'www. my-sinusitis-coach.com/data' contains a QR code that can be scanned by the patient with his/her smartphone. At that time, the patient authorizes visualization of his/her data on the PC. Scanning allows the physician to identify the specific patient, to evaluate disease control and medication use; and discuss this information with the patient to determine or adapt CRS treatment for the following period.

\section{Measuring quality of life}

\section{SNOT-22 questionnaire}

The SinoNasal Outcome Test (SNOT)-22 questionnaire is widely used and validated to assess quality of life in CRS patients, to evaluate treatment effectiveness or to identify patients eligible for sinus surgery ${ }^{(23-26)}$. This questionnaire has been included in the app as an optional questionnaire to complete monthly.

\section{EQ-5D-5L questionnaire}

The EuroQol-5Dimension-5Level (EQ-5D-5L) generic question- naire is used to assess the general health-related quality of life of chronically ill patients ${ }^{(27)}$. Five specific dimensions of the patients' health are scored: mobility, self-care, usual activities, pain/ discomfort and anxiety/depression. Such generic questionnaire allows comparison of the patients' overall health across different medical conditions. Similar to the SNOT-22 questionnaire, the EQ-5D-5L questionnaire has been included in the app as an optional questionnaire to complete monthly.

\section{Patient support through reliable education}

Patients are in need of information about their disease and treatment options. Their needs may vary depending on their phase in the disease journey but often the internet is their primary source of information (unpublished data). This information might however be biased or unreliable. Consequently, EUFOREA has created a support platform for patients with upper airway disease with both online educational material as well as the educational platform available from mySinusitisCoach. EUFOREA key opinion leaders (KOLs) have collected frequently asked questions from their patients and answered them in short and easy to understand language. The questions and answers (Q\&A) have been checked to match the health literacy of the average patient by a panel of primary care physicians for each country. The Q\&A were classified into 5 topics (disease, diagnosis, treatment, surgery and quality of life) to facilitate the search for the patients.

\section{Expected benefits for the different stakeholders}

For the patient

This tool was developed in collaboration with patients to ensure acceptability and use over a prolonged period of time by a large group of patients. Feedback by the European patient organization (EFA) was obtained at different time points and the app was tested with patients during the development phase. Close monitoring of the patients' rhinosinusitis symptoms, feedback about disease control and advice to contact their treating physician may lead to increased awareness of the patients about their disease, thereby contributing to better compliance with their therapy. The educational platform may lead to better knowledge about CRS and available treatment options allowing active participation of patients in the management of their disease.

\section{For the healthcare professional}

Longitudinal information about disease control and adherence to therapy will be available for the doctor. This information may help to decide upon the best treatment strategy and facilitate the patient-doctor interaction ${ }^{(28)}$. The educational platform may aid the doctor in providing information on treatment options to the patient or allow referral to the platform which might be time saving. In addition this tool could identify those patients who are in need of more extensive medical or surgical treatment ${ }^{(29)}$. 
In the post-operative setting it may facilitate the follow-up of the surgical intervention as well as duration of effect.

\section{For the scientific community}

Currently, guidelines and care pathways are developed based on clinical studies that are performed in highly selected patient populations, for valid scientific reasons, however this does not validate the extrapolation of results achieved into the general population ${ }^{(30)}$. mySinusitisCoach will provide real life data on the natural course of disease, socio-economic impact of CRS and outcomes of medical as well as surgical interventions. In addition, it allows the evaluation of the impact of CRS on lower airway symptoms, sleep quality and work productivity.

\section{Authorship contribution}

SS, BP, JB, CB, WJF and PWH developed mySinusitisCoach, wrote the manuscript and reviewed the manuscript. Others: provided feedback on design, development and implementation of the app during brainstorming sessions; and reviewed the manuscript.

\section{Conflict of interest}

No conflict of interest.

\section{References}

1. Hastan D, Fokkens WJ, Bachert C, Newson RB, Bislimovska J, Bockelbrink A, et al. Chronic rhinosinusitis in Europe--an underestimated disease. A GA2LEN study. Allergy. 2011:66(9):1216-23.

2. Smith KA, Orlandi RR, Rudmik L. Cost of adult chronic rhinosinusitis: A systematic review. The Laryngoscope. 2015;125(7):1547-56.

3. De Greve G, Hellings PW, Fokkens WJ, Pugin B, Steelant B, Seys SF. Endotype-driven treatment in chronic upper airway diseases. Clin Transl Allergy. 2017;7:22.

4. Fokkens WJ, Lund VJ, Mullol J, Bachert C, Alobid I, Baroody F, et al. EPOS 2012: European position paper on rhinosinusitis and nasal polyps 2012. A summary for otorhinolaryngologists. Rhinology. 2012;50(1):1-12.

5. van der Veen J, Seys SF, Timmermans $M$

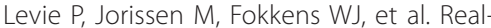
life study showing uncontrolled rhinosinusitis after sinus surgery in a tertiary referral centre. Allergy. 2017;72(2):282-290.

6. Snidvongs K, Heller GZ, Sacks R, Harvey RJ. Validity of European Position Paper on Rhinosinusitis Disease Control Assessment and Modifications in Chronic Rhinosinusitis. Otolaryngol-Head Neck Surg. 2014:150(3):479-86.

7. Hellings PW, Fokkens WJ, Akdis C, Bachert C Cingi C, Dietz de Loos D, et al. Uncontrolled allergic rhinitis and chronic rhinosinusitis: where do we stand today? Allergy. 2013;68(1):1-7.

8. Hastan D, Fokkens WJ, Bachert C, Newson RB, Bislimovska J, Bockelbrink A, et al. Chronic rhinosinusitis in Europe--an underestimated disease. A GA2LEN study. Allergy. 2011;66(9):1216-23.

9. Hellings PW, Fokkens WJ, Bachert C, Akdis CA, Bieber T, Agache I, et al. Positioning the Principles of Precision Medicine in Care Pathways for Allergic Rhinitis and Chronic Rhinosinusitis - an EUFOREA-ARIAEPOS-AIRWAYS ICP statement. Allergy. 2017;72(9):1297-1305

10. mHealth app developer economics study [Internet]. 2016. Available from: https://
research2guidance.com/r2g/r2g-mHealthApp-Developer-Economics-2016.pdf

11. Bousquet J, Caimmi DP, Bedbrook A, Bewick M, Hellings PW, Devillier $P$, et al. Pilot study of mobile phone technology in allergic rhinitis in European countries: the MASKrhinitis study. Allergy. 2017;72(6):857-65

12. Bousquet J, Schünemann $\mathrm{HJ}$, Hellings $\mathrm{PW}$, Arnavielhe S, Bachert C, Bedbrook A, et al. MACVIA clinical decision algorithm in adolescents and adults with allergic rhinitis. Allergy Clin Immunol. 2016;138(2):367-374. e2.

13. Bousquet J, Caimmi DP, Bedbrook A, Bewick M, Hellings PW, Devillier P, et al. Pilot study of mobile phone technology in allergic rhinitis in European countries: the MASKrhinitis study. Allergy. 2017:72(6):857-65.

14. Bousquet J, Bewick M, Arnavielhe S, Mathieu-Dupas E, Murray R, Bedbrook A, et al. Work productivity in rhinitis using cell phones: The MASK pilot study. Allergy. 2017;72(10):1475-84.

15. Caimmi D, Baiz N, Tanno LK, Demoly P, Arnavielhe S, Murray R, et al. Validation of the MASK-rhinitis visual analogue scale on smartphone screens to assess allergic rhinitis control. Clin Exp Allergy. 2017:42(12):1526-1533.

16. Muraro A, Fokkens WJ, Pietikainen S, Borrelli D, Agache I, Bousquet J, et al. European symposium on precision medicine in allergy and airways diseases: report of the European Union parliament symposium (October 14, 2015). Rhinology. 2015;53(4):303-7.

17. Muraro A, Lemanske RF, Hellings PW Akdis CA, Bieber T, Casale TB, et al. Precision medicine in patients with allergic diseases: Airway diseases and atopic dermatitis-PRACTALL document of the European Academy of Allergy and Clinical Immunology and the American Academy of Allergy, Asthma \& Immunology. J Allergy Clin Immunol. 2016;137(5):1347-58.

18. Hellings PW, Akdis CA, Bachert C, Bousquet J, Pugin B, Adriaensen $G$, et al. EUFOREA Rhinology Research Forum 2016: report of the brainstorming sessions on needs and priorities in rhinitis and rhinosinusitis.
Rhinology. 2017:55(3):202-10.

19. Philpott C, Erskine S, Smith R, Hopkins C, Kara N, Farboud A, et al. Current use of baseline medical treatment in chronic rhinosinusitis: Data from the National Chronic Rhinosinusitis Epidemiology Study (CRES). Clin Otolaryngol. 2017; doi: 10.1111/ coa.13012. [Epub ahead of print]

20. Rudmik L, Xu Y, Liu M, Bird C, Kukec E, Quan H. Utilization Patterns of Topical Intranasal Steroid Therapy for Chronic Rhinosinusitis: A Canadian Population-Based Analysis. JAMA Otolaryngol-- Head Neck Surg. 2016;142(11):1056-62.

21. Nabi S, Rotenberg BW, Vukin I, Payton K, Bureau Y. Nasal spray adherence after sinus surgery: problems and predictors. J Otolaryngol - Head Neck Surg. 2012;41 Suppl 1:S49-55.

22. Bousquet J, Schunemann HJ, Fonseca J, Samolinski B, Bachert C, Canonica GW, et al. MACVIA-ARIA Sentinel Network for allergic rhinitis (MASK-rhinitis): the new generation guideline implementation. Allergy. 2015;70(11):1372-92.

23. Piccirillo JF, Merritt MG, Richards ML. Psychometric and clinimetric validity of the 20-Item Sino-Nasal Outcome Test (SNOT-20). Otolaryngol--Head Neck Surg. 2002;126(1):41-7.

24. Hopkins C, Gillett S, Slack R, Lund VJ, Browne JP. Psychometric validity of the 22-item Sinonasal Outcome Test. Clin Otolaryngol. 2009;34(5):447-54.

25. Dietz de Loos DAE, Segboer CL, Gevorgyan A, Fokkens WJ. Disease-specific quality-oflife questionnaires in rhinitis and rhinosinusitis: review and evaluation. Curr Allergy Asthma Rep. 2013;13(2):162-70.

26. Rudmik L, Soler ZM, Hopkins C, Schlosser RJ, Peters A, White AA, et al. Defining appropriateness criteria for endoscopic sinus surgery during management of uncomplicated adult chronic rhinosinusitis: a RAND/ UCLA appropriateness study. Rhinology. 2016;54(2):117-28.

27. Herdman M, Gudex C, Lloyd A, Janssen M Kind P, Parkin D, et al. Development and preliminary testing of the new five-level version of EQ-5D (EQ-5D-5L). Qual Life Res. 
2011;20(10):1727-36.

28. Gamble J, Stevenson M, Heaney LG. A study of a multi-level intervention to improve non-adherence in difficult to control asthma. Respir Med. 2011;105(9):1308-15.

29. Rudmik L, Soler ZM, Hopkins C. Using postoperative SNOT-22 to help predict the probability of revision sinus surgery. Rhinology. 2016;54(2):111-6.

30. Costa DJ, Amouyal M, Lambert P, Ryan D, Schünemann $\mathrm{HJ}$, Daures JP, et al. How rep- resentative are clinical study patients with allergic rhinitis in primary care? J Allergy Clin Immunol. 2011;127(4):920-926.e1.

Sven Seys

EUFOREA

Brand Whitlocklaan 132

1300 Brussels

Belgium

E-mail: sven.seys@euforea.eu 